\title{
NEAR PERMUTATION SEMIGROUPS
}

\author{
JORGE M. ANDRÉ* \\ Centro de Álgebra da Universidade de Lisboa, \\ Av. Prof. Gama Pinto, 2, \\ 1649-003 Lisboa, Portugal \\ and \\ Departamento de Matemática \\ Faculdade de Ciências e Tecnologia da Universidade Nova de Lisboa \\ Monte da Caparica, 2829-516 Caparica, Portugal \\ E-mail: jmla@fct.unl.pt
}

\begin{abstract}
A transformation semigroup over a set $X$ with $N$ elements is said to be a near permutation semigroup if it is generated by a group of permutations on $N$ elements and by a set of transformations of rank $N-1$. In this paper we make a summary of the author's thesis, in which are exhibited ways of checking whether those specific transformation semigroups belong to various structural classes of semigroups, just by analysis of the generators and without first having to generate them.
\end{abstract}

\section{Introduction}

In this paper we make a summary of the more important results of the author's thesis [6] about near permutation semigroups $S=\langle G, \mathcal{U}\rangle$, that is semigroups generated by a group $G$ of permutations on $N$ elements, for some $N \in \mathbb{N}$, and by a set $\mathcal{U}$ of transformations of rank $N-1$. It is known that the number of elements of the transformation semigroups on a set $X$ with a finite number $N$ of elements, grows exponentially with the cardinality of the set $X$. We aim to find efficient ways to determine the structure of a given near permutation semigroup, without first having to generate it. The motivation for the study of those semigroups is due to the following. The semigroup $\mathcal{T}_{N}$ of all full transformations on a set $X$ with $N$ elements, for some $N \in \mathbb{N}$, is generated by the the group of all permutations on the set $X$ and by any idempotent of $\operatorname{rank} N-1$. Similarly

*Work partially supported by the Sub-programa Ciência e Tecnologia do $2^{\circ}$ Quadro Comunitário de Apoio (grant number BD/18012/98) and project POCTI/32440/MAT/2000 (CAUL) of FCT and FEDER. 
the symmetric inverse semigroup $\mathcal{I}_{N}$ on $X$, that is the semigroup of all injective partial transformations on a set with $N$ elements, is generated by its group of units and by any idempotent of $\operatorname{rank} N-1$. For the two previous results refer to [8]. Other well known semigroups like the semigroup $\mathcal{O} \mathcal{P}_{N}$ of all orientation preserving mappings and the semigroup $\mathcal{O} \mathcal{P} \mathcal{R}_{N}$ of all orientation preserving and reversing mappings, on a finite chain of order $N$, are generated by a group of permutations and by an idempotent of rank $N-1$ (see [7]). Moreover, McAlister [12] proved that all semigroups generated by a group of permutations on $N$ elements and by an idempotent of rank $N-1$ are regular. He also obtained necessary and sufficient conditions for a transformation semigroup generated by a group of permutations on $N$ elements and by an idempotent of rank $N-1$ to be inverse. In our work of [3] and [1], we considered a set $\mathcal{U}$ of transformations of $\operatorname{rank} N-1$ instead of the idempotent $e$.

To obtain most of the results the author made use of computational tools such as the PC software [9] and [11].

\section{Preliminaries}

Let $X=\{1,2, \ldots, N\}$, where $N \in \mathbb{N}$. As usual, we denote by $\mathcal{T}_{N}$ the monoid of all full transformations of $X$ (under composition), and by $\mathcal{S}_{N}$ the symmetric group on $X$. We call a semigroup generated by a group of permutations on $N$ elements and by a set of transformations of rank $N-1$ a near permutation semigroup. Denote by $\left(\begin{array}{l}x \\ y\end{array}\right)$, where $x, y \in X$, the idempotent of $\mathcal{T}_{N}$ which maps $x$ to $y$ and $i$ to itself for $i \neq x$. An useful observation is that an element $a$ belongs to a subgroup of $\mathcal{T}_{N}$ if and only if $\operatorname{rank} a=\operatorname{rank} a^{2}$. Given $z \in \mathcal{T}_{N}$ denote by $\operatorname{Im} z$ the image set of the transformation $z$. It is also useful to note that given an element $e$ of $\mathcal{T}_{N}$, then $e$ is idempotent if and only if it fixes all elements of $\operatorname{Im} e$. Given $G$ a group of permutations on $X$ and $x \in X$ define the sets $O_{G}(x)=\{y \in X$ : $y=x g$, for some $g \in G\}$ and $\operatorname{Stab}_{G}(x)=\{g \in G: x g=x\}$, which we also call the orbit of $x$ under $G$ and the stabilizer of $x$ under $G$, respectively.

A directed graph $\Gamma$ consists of two sets, a non empty set $V(\Gamma)$ of vertices and a set $A(\Gamma)$ of arrows of $\Gamma$, together with a mapping $\Psi: A(\Gamma) \longrightarrow$ $V(\Gamma) \times V(\Gamma)$.

Given $a \in A(\Gamma)$ and $a \Psi=\left(v_{1}, v_{2}\right)$, where $v_{1}, v_{2} \in V(\Gamma)$, we call $v_{1}$ and $v_{2}$ the initial vertex and the terminal vertex, respectively. We say that $a$ is an arrow from $v_{1}$ to $v_{2}$.

A path between two vertices $v_{1}$ and $v_{2}$, in a graph $\Gamma$, is a sequence of 
arrows such that the terminal vertex of one arrow is the initial vertex of the next and the first arrow of the sequence has initial vertex $v_{1}$ and the last arrow has terminal vertex $v_{2}$.

Let $S$ be a near permutation semigroup and $u$ be a transformation in $S$ of rank $N-1$. We denote by $\alpha(u)$ and $\beta(u)$ the two unique distinct elements $a, b \in X$ such that $(a, b) \in \operatorname{Ker} u$. We denote by $\gamma(u)$ the unique element of $X \backslash I m u$. If $u$ is being denoted by $u_{i}$ for some $i \in\{1, \ldots, m\}$, we abbreviate $\alpha(u), \beta(u)$ and $\gamma(u)$, respectively, by $\alpha_{i}, \beta_{i}$ and $\gamma_{i}$. Given $(a, b) \in \operatorname{Ker} u$, with $a \neq b$, in order to choose which one $a$ or $b$ is $\alpha(u)$, we set $\alpha(u)$ as the minimum of the set $\{a, b\}$, for each $u \in \mathcal{U}$.

\section{Regular near permutation semigroups}

Let $S=\langle G, \mathcal{U}\rangle$ be a near permutation semigroup and $\mathcal{U}=\left\{u_{1}, \ldots, u_{m}\right\}$. Consider the directed graph $\Gamma(G ; \mathcal{U})$, where the vertices are the elements of $\mathcal{U}$ and there is an arrow, $u_{i} \rightarrow u_{j}$ between two vertices $u_{i}$ and $u_{j}$, if $\gamma_{i} g \in\left\{\alpha_{j}, \beta_{j}\right\}$. Notice that given $u_{i}, u_{j} \in \mathcal{U}$, the existence of $u_{i} \rightarrow u_{j}$ is equivalent to saying that rank $u_{i} g u_{j}=N-1$, for some $g \in G$. In that case we can also refer that property by writing $u_{i} \rightarrow_{g} u_{j}$. In the graph $\Gamma(G ; \mathcal{U})$, represent each vertex $u_{i}$, where $i=1, \ldots, m$, with the triple $\left(\alpha_{i}, \beta_{i}, \gamma_{i}\right)$, where $\left(\alpha_{i}, \beta_{i}\right) \in \operatorname{Ker} u_{i}, \alpha_{i}<\beta_{i}$ and $\gamma_{i} \in X \backslash \operatorname{Im} u_{i}$. We call $\Gamma(G ; \mathcal{U})$ a near permutation graph of $S$. For these defined graphs, if we consider more than one vertex representing the same $\mathcal{H}$-class, we obtain equivalent graphs to the ones where there is only one vertex for each $\mathcal{H}$-class in the sense that both graphs give us the same information for our study. Thus, when we consider the graph $\Gamma(G ; \mathcal{U})$ of a near permutation semigroup $S$, we only include in the set of vertices the elements of $\mathcal{U}$ that belong to different $\mathcal{H}$-classes of $\mathcal{T}_{N}$. Hence we choose exactly one vertex, from the vertices that have the same triples $\left(\alpha_{i}, \beta_{i}, \gamma_{i}\right)$.

Note that in the graph $\Gamma(G ; \mathcal{U})$ there exists at most one arrow between two vertices.

A special case occurs when $\mathcal{U}$ is a group. In this case, the graph $\Gamma(G ; \mathcal{U})$ is complete, that is there exists exactly one arrow between any two distinct vertices.

Let $\Gamma(G ; \mathcal{U})$ be the near permutation graph of a near permutation semigroup $S=\langle G, \mathcal{U}\rangle$. Then there exists a binary relation $\varepsilon$ on $\mathcal{U}$ induced by the graph $\Gamma(G ; \mathcal{U})$, defined by: given $v_{0}, v_{0}^{\prime} \in \mathcal{U}$,

$v_{0} \varepsilon v_{0}^{\prime} \quad$ if and only if there exists a path $v_{0} \rightarrow v_{1} \rightarrow v_{2} \rightarrow \ldots \rightarrow v_{k} \rightarrow v_{0}^{\prime}$

in $\Gamma(G ; \mathcal{U})$, for some $v_{1}, v_{2}, \ldots, v_{k} \in \mathcal{U}$. 
Clearly the relation $\varepsilon$ is transitive. Note also that the existence of a path $v_{1} \rightarrow_{g_{1}} v_{2} \rightarrow g_{2} \ldots v_{l-1} \rightarrow g_{l-1} v_{l}$ in $\Gamma(G ; \mathcal{U})$, is equivalent to the equality rank $v_{1} g_{1} v_{2} \ldots g_{l-1} v_{l}=N-1$.

Observe that, given $u, v \in \mathcal{U}$ and $x \in S$, if rank $u x v=N-1$ then exists a path, relative to the graph $\Gamma(G ; \mathcal{U})$, from $u$ to $v$.

The first two results show us that we can use the near permutation graph of a semigroup $S$ in order to determine whether the generators or the elements of $S$ with rank $N-1$ are regular.

Proposition 3.1. [3] Let $S=\langle G, \mathcal{U}\rangle$ be a near permutation semigroup. Then, the elements of $\mathcal{U}$ are regular if and only if the relation $\varepsilon$ is reflexive.

Theorem 3.1. [3] Let $S=\langle G, \mathcal{U}\rangle$ be a near permutation semigroup. Then the elements of $S$ with rank $N-1$ are regular if and only if the relation $\varepsilon$, induced by the graph $\Gamma(G ; \mathcal{U})$, is an equivalence relation.

Before determining a characterization of the idempotents of rank $N-1$, we need some more notation. For each $u_{i} \in \mathcal{U}$, we fix a $u_{i}^{\prime}$ an inverse of $u_{i}$ in $S$, and consider $u_{i} u_{i}^{\prime}=\left(\begin{array}{c}x_{i} \\ y_{i}\end{array}\right)$, where $\left\{x_{i}, y_{i}\right\}=\left\{\alpha_{i}, \beta_{i}\right\}$, for $i=1, \ldots, m$. We also define $T=\left\{\left(\begin{array}{l}x_{1} \\ y_{1}\end{array}\right), \ldots,\left(\begin{array}{l}x_{m} \\ y_{m}\end{array}\right)\right\}$ and $T^{\prime}=\left\{\left(\begin{array}{c}\beta \\ \alpha\end{array}\right) \in S:\left(\begin{array}{c}\alpha \\ \beta\end{array}\right) \in T\right\}$.

According to the previous definitions, it is important to know when both idempotents $\left(\begin{array}{c}\alpha_{i} \\ \beta_{i}\end{array}\right)$ and $\left(\begin{array}{c}\beta_{i} \\ \alpha_{i}\end{array}\right)$ belong to $S$. The next proposition gives an answer to this question and in particular tells us how to determine the set $T^{\prime}$ for each semigroup $S$.

Proposition 3.2. [3] Let $S=\langle G, \mathcal{U}\rangle$ be a near permutation semigroup such that the relation $\varepsilon$ induced by the graph $\Gamma(G ; \mathcal{U})$ is an equivalence relation. Then, for each $i \in\{1,2, \ldots, m\}$, the idempotents $\left(\begin{array}{c}\alpha_{i} \\ \beta_{i}\end{array}\right)$ and $\left(\begin{array}{c}\beta_{i} \\ \alpha_{i}\end{array}\right)$ belong to $S$ if and only if $\left\{\alpha_{i}, \beta_{i}\right\}=\left\{\gamma_{p} g_{1}, \gamma_{q} g_{2}\right\}$, for some $g_{1}, g_{2} \in G$ and $u_{p}, u_{q} \in \mathcal{U}$.

Now we can easily determine the rank $N-1$ idempotents of $S$.

Corollary 3.1. [3] Let $S=\langle G, \mathcal{U}\rangle$ be a near permutation semigroup. Then the set of idempotents of $S$ with rank $N-1$ is $\bigcup_{g \in G} g^{-1}\left(T \cup T^{\prime}\right) g$.

The Proposition 3.2 is very important in order to obtain an easy to check sufficient condition for $S$ to be regular.

Theorem 3.2. [3] Let $S=\langle G, \mathcal{U}\rangle$ be a near permutation semigroup. If the relation $\varepsilon$ induced by the graph $\Gamma(G ; \mathcal{U})$ is an equivalence relation and, for each $i \in\{1, \ldots, m\}$, there exist $g_{i}, g_{i}^{\prime} \in G$ and $u_{p}, u_{q} \in \mathcal{U}$, such that $\left\{\alpha_{i}, \beta_{i}\right\}=\left\{\gamma_{p} g_{i}, \gamma_{q} g_{i}^{\prime}\right\}$, then $S$ is regular. 
Now we present some more useful and efficient sufficient conditions for $S$ to be regular which do not involve the determination of the idempotents of $S$ with $\operatorname{rank} N-1$.

Theorem 3.3. [3] Let $S=\langle G, \mathcal{U}\rangle$ be a near permutation semigroup. If for each $i \in\{1, \ldots, m\}$, the elements $\alpha_{i}, \beta_{i}$ and $\gamma_{i}$ belong to the same orbit under $G$, then $S$ is regular.

The next sufficient condition is a particular case of the previous and says that if for any two elements $x$ and $y$ of $X$ exists $g \in G$ such that $x g=y$ then $S$ is regular.

Theorem 3.4. [3] Let $S=\langle G, \mathcal{U}\rangle$ be a near permutation semigroup. If $G$ acts transitively on $X$, then $S$ is regular.

Now we have another condition for the study of the regularity of $S$ which is independent of the previous and also very useful in some cases.

Theorem 3.5. [3] Let $S=\langle G, \mathcal{U}\rangle$ be a near permutation semigroup. If the relation $\varepsilon$ induced by the graph $\Gamma(G ; \mathcal{U})$ is reflexive and, for each $i \in$ $\{1, \ldots, m\}$, the elements $\alpha_{i}$ and $\beta_{i}$ belong to the same orbit under $G$ then $S$ is regular.

Next we have a theorem which provides a more general sufficient condition for the study of the regularity of $S$, which turned out to be also necessary in all the examples tested.

Theorem 3.6. [3] Let $S=\langle G, \mathcal{U}\rangle$ be a near permutation semigroup. Suppose that the relation $\varepsilon$ induced by the graph $\Gamma(G ; \mathcal{U})$ is an equivalence relation. If for all $\left(\begin{array}{c}\alpha_{k} \\ \beta_{k}\end{array}\right) \in T$ such that $\left(\begin{array}{c}\beta_{k} \\ \alpha_{k}\end{array}\right) \notin T \cup T^{\prime}$ and for all $\gamma_{s}$ g which are different from $\alpha_{k}$ and $\beta_{k}$, with $s \in\{1, \ldots, m\}$ and $g \in G$, there exist $g^{\prime} \in G$ and $\left(\begin{array}{l}a \\ b\end{array}\right) \in T \cup T^{\prime}$ satisfying:

i) $\gamma_{s} g=a g^{\prime}$;

ii) $\alpha_{k}$ belongs to the orbit of $\beta_{k}$ under the group $H_{\left(\begin{array}{c}a g^{\prime} \\ b g^{\prime}\end{array}\right)}$;

iii) $b g^{\prime} \neq \beta_{k}$;

then $S$ is regular.

The following theorem shows that the study of the regularity of $S$, when $\mathcal{U}$ is a set of idempotents, depends only on its near permutation graph. 
Theorem 3.7. [3] Let $S=\langle G, E\rangle$ be a near permutation semigroup, where $E$ is a set of idempotents. Then $S$ is regular if and only if the relation $\varepsilon$ induced by the graph $\Gamma(G ; E)$ is an equivalence relation.

In [10] was obtained a necessary and sufficient condition for a semigroup generated by a group of permutations on a set with $N$ elements and by a set of idempotents of rank $N-1$, to contain all singular transformations of $\mathcal{T}_{N}$. With the use of the tools and results obtained in [3] we have a different approach to that problem, in which case the semigroup is generated by a group of permutations and by a set of $\operatorname{rank} N-1$ transformations.

But first we need to define several sets. Let be $\mathcal{U}=\left\{u_{1}, \ldots, u_{m}\right\}$ and $I=\{1, \ldots, m\}$. Let also be $A=\{\{x, y\}: x, y \in X, x \neq y\}$, $B=\left\{\left\{\alpha_{i}, \beta_{i}\right\}: i \in I\right\}, C=\left\{a \in X \backslash\right.$ Im $\left.u_{i}: i \in I\right\}$ and $D=\left\{\alpha_{i}: i \in I\right\} \cup\left\{\beta_{i}: i \in I\right\}$. Now, the group $G$ acts on the sets $B$ and $C$ (on the right), in the following ways:

$$
B G=\left\{\left\{\alpha_{i} g, \beta_{i} g\right\}: i \in I, g \in G\right\} \text { and } C G=\{a g: a \in C, g \in G\} .
$$

The proof of the theorem is in [4].

Theorem 3.8. Let $S=\langle G, \mathcal{U}\rangle$ be a near permutation semigroup. Then $S$ contains $\mathcal{T}_{N} \backslash \mathcal{S}_{N}$ if and only if

1) The relation $\varepsilon$ is an equivalence relation;

2) $B G=A$;

3) $D \subseteq C G$.

\section{Near permutation semigroups generated by two groups}

An interesting special case of the general case considered previously is when $\mathcal{U}$ is a group. In fact, in the case considered by McAlister in [12], the set $\mathcal{U}$ has only one element, which is an idempotent. Thus it is a group.

Let $S=\langle G, H\rangle$ be a near permutation semigroup, where $H$ is a group. We can rearrange the set $X$ so that the identity of $H$ is $e=\left(\begin{array}{l}1 \\ 2\end{array}\right)$.

From now on, given $S=\langle G, H\rangle$ a near permutation semigroup, where $H$ is a group, let $e=\left(\begin{array}{l}1 \\ 2\end{array}\right)$ be the identity of $H$.

The assumption that $2 \in O_{G}(1)$ is crucial in the study of the case $\mathcal{U}=\{e\}$. This was shown by McAlister in [12].

Theorem 4.1. Let $S=\langle G, e\rangle$ be a near permutation semigroup, where $e$ is an idempotent. If $2 \notin O_{G}(1)$, then $S$ is an $\mathcal{R}$-unipotent semigroup.

It was also proved in [12] that $S=\langle G, e\rangle$ is always a regular semigroup. 
Theorem 4.2. Let $S=\langle G, e\rangle$ be a near permutation semigroup, where e is an idempotent. Then $S$ is regular.

The knowledge of the orbit of 1 under $G$ is also very important in the more general case, where $S=\langle G, \mathcal{U}\rangle$, as the following results show.

Theorem 4.3. [3] Let $S=\langle G, H\rangle$ be a near permutation semigroup, where $H$ is a group. If $2 \in O_{G}(1)$, then $S$ is a regular semigroup.

The next result is obtained by considering a near permutation semigroup generated by a group and by an idempotent and which contains $S$. But first we need to define the following. Let $S=\langle G, H\rangle$ be a near permutation semigroup, where $H$ is a group with identity $\left(\begin{array}{l}1 \\ 2\end{array}\right)$. Since the elements of $H$ are group elements of $\operatorname{rank} N-1$, and every element $h$ of the group $H$ satisfies $1 \notin \operatorname{Im} h$ and $(1,2) \in K e r h$, we can associate to each $h \in H$ a permutation $\bar{h}$ such that $1 \bar{h}=1$ and that $x \bar{h}=x h$, for all $x \in X \backslash\{1\}$. From now on, we denote by $\bar{h}$ the corresponding unique permutation of the transformation $h \in H$. The set of all such elements $\bar{h}$ is a group of permutations, that we denote by $\bar{H}$. The semigroup $\langle G, \bar{H}\rangle$ is a group, because all its generators are permutations. We denote $\langle G, \bar{H}\rangle$ by $G(S)$ and the set $\{g \in G: 1 g \neq 1\}$ by $G^{\prime}$. Observe that $S=\langle G, e \bar{H}\rangle$ and $S$ is contained in the semigroup $\langle G(S), e\rangle$. Conversely if $G$ and $W$ are subgroups of $\mathcal{S}_{N}$ such that the elements of $W$ fix 1 , that is $1 w=1$ for all $w \in W$, then $S=\langle G, e W\rangle$ is a near permutation semigroup, where $e W$ is a group of transformations of $\operatorname{rank} N-1$. Now, we have the semigroup $S=\langle G, H\rangle$ between two semigroups generated by a group and an idempotent, that is $S^{\prime}=\langle G, e\rangle \subseteq S \subseteq\langle G(S), e\rangle$.

Proposition 4.1. [3] Let $S=\langle G, H\rangle$ be a near permutation semigroup, where $H$ is a group. If $2 \notin O_{G(S)}(1)$, then $S$ is an $\mathcal{R}$-unipotent semigroup.

To study a near permutation semigroup $S=\langle G, H\rangle$, it will be very important to know some results about the local submonoid $e S e$, where $e$ is the identity of $H$. Consider $H_{e}$ as being the $\mathcal{H}$-class of $e$.

Proposition 4.2. [3] Let $S=\langle G, H\rangle$ be a near permutation semigroup, where $H$ is a group with identity e. If $2 \notin O_{G}(1)$, then eSe is generated by $H_{e}$ together with the set $\{$ ege $: g \in G, 1 g \neq 1\}$.

Moreover, the group $H_{e}$ is generated by $H$ together with the set ege: $g \in G, 1 g=1\}$. 
In order to use the results of the previous section it is important the following result.

Proposition 4.3. [3] Let $S=\langle G, H\rangle$ be a near permutation semigroup, with $H$ a group. Suppose that $2 \notin O_{G}(1)$. Then there exists an isomorphism $\psi$ between the submonoid eSe of $S$ and a submonoid of $\mathcal{T}_{\{2, \ldots, N\}}$, which has as group of units $H_{e} \psi$. Moreover, eSe is isomorphic to the near permutation semigroup $W_{S}=\left\langle H_{e} \psi, V \psi\right\rangle$, where $H_{e}$ is the $\mathcal{H}$-class of $e$ and $V=\{g \in$ $G: 1 g \neq 1\}$.

Using the isomorphic copy of the local submonoid $e S e$, we can say that $e S e$ is a near permutation semigroup $\left\langle G_{S}, \mathcal{U}_{S}\right\rangle$, where $G_{S}=H_{e} \psi$ and $\mathcal{U}_{S}=$ $V \psi$. We recall that it is convenient to consider $\mathcal{U}_{S}=\left\{u_{1}, \ldots, u_{m}\right\}$ and $I=\{1, \ldots, m\}$. Now we can refer some more results about the regularity of $S$ with the help of the technique described before.

Proposition 4.4. [3] Let $S=\langle G, H\rangle$ be a near permutation semigroup, where $H$ is a group and $W_{S}=\left\langle G_{S}, \mathcal{U}_{S}\right\rangle$. If, for each $i \in I$, the elements $\alpha_{i}, \beta_{i}$ and $\gamma_{i}$ belong to the same orbit under $G_{S}$, then $S$ is regular.

According to the results of the previous section is easy to conclude that all rank $N-1$ elements of $S$ are regular. Next we provide a tool for checking if the elements of rank $N-2$ are regular, which is obtained using Theorem 3.1 on the local submonoid $e S e$ of $S$.

Theorem 4.4. [3] Let $S=\langle G, H\rangle$ be a near permutation semigroup, where $H$ is a group and $W_{S}=\left\langle G_{S}, \mathcal{U}_{S}\right\rangle$, as defined above. Then the elements of $S$ with rank $N-2$ are regular if and only if the relation $\varepsilon$ induced by the graph $\Gamma\left(G_{S} ; \mathcal{U}_{S}\right)$, where the vertices are the elements of the set $\mathcal{U}_{S}=\left\{u_{1}, \ldots, u_{m}\right\}$, is an equivalence relation.

We have one more sufficient condition for $S$ to be regular which uses Theorem 3.5.

Proposition 4.5. [3] Let $S=\langle G, H\rangle$ be a near permutation semigroup, where $H$ is a group and $W_{S}=\left\langle G_{S}, \mathcal{U}_{S}\right\rangle$. If the relation $\varepsilon$ induced by the graph $\Gamma\left(G ; \mathcal{U}_{S}\right)$ is an equivalence relation and, for each $i \in I$, the elements $\alpha_{i}$ and $\beta_{i}$ belong to the same orbit under $G_{S}$, then $S$ is regular.

It is important to observe that, given a near permutation semigroup $S=\langle G, H\rangle$, we can use the more general result of Theorem 3.6, considering the local submonoid $e S e$ of $S$ and its isomorphic copy $W_{S}=\left\langle G_{S}, \mathcal{U}_{S}\right\rangle$. 
The results that follow are about inverse near permutation semigroups. The first one has to do with the semigroup $\langle G, e\rangle$ and was proved in [12].

Theorem 4.5. Let $S=\langle G, H\rangle$ be a near permutation semigroup, where $H$ is a group with identity $e$. The subsemigroup $S^{\prime}=\langle G, e\rangle$ of $S$ is inverse if and only if $2 \notin O_{G}(1)$ and $\operatorname{Stab}_{G}(1) \subseteq \operatorname{Stab}_{G}(2)$.

With the group $G(S)$ previously defined it is possible to say more about when $S$ is inverse. Next, we obtain a sufficient but not necessary condition for $S$ to be inverse.

Proposition 4.6. [1] Let $S=\langle G, H\rangle$ be a near permutation semigroup, where $H$ is a group with identity e. If $2 \notin O_{G(S)}(1)$ and $\operatorname{Stab}_{G(S)}(1) \subseteq$ $\operatorname{Stab}_{G(S)}(2)$, then $S$ is inverse.

It follows a theorem that provides a necessary and sufficient condition for $S=\langle G, H\rangle$ to be inverse. Recall that $G(S)=\langle G, \bar{H}\rangle$, where $\bar{H}$ is the group of permutations associated with $H$.

Theorem 4.6. [1] Let $S=\langle G, H\rangle$ be a near permutation semigroup, where $H$ is a group. Then $S$ is inverse if and only if

(i) $2 \notin O_{G(S)}(1)$;

(ii) If $1 g_{1} \bar{h}_{1} \ldots g_{n-1} \bar{h}_{n-1} g_{n}=1$ and $1 g_{1} \bar{h}_{1} \ldots g_{s-1} \bar{h}_{s-1} g_{s} \neq 1$, for all $s$ such that $1<s<n$, then $2 g_{1} \bar{h}_{1} \ldots g_{n-1} \bar{h}_{n-1} g_{n}=2$, where $g_{1}, \ldots, g_{n} \in G$ and $\bar{h}_{1}, \ldots, \bar{h}_{n-1} \in \bar{H}$.

Notice that Theorem 4.5, proved by McAlister in [12], is an easy consequence of Theorem 4.6. Moreover, it is obvious that if $\operatorname{Stab}_{G(S)}(1) \subseteq$ $\operatorname{Stab}_{G(S)}(2)$ then $2 h=2$, for all $h \in H$.

Observe also that the conditions of Theorem 4.6 can be simplified when, for $S=\langle G, H\rangle$, the elements of $H$ satisfy the equality $2 h=2$.

Corollary 4.1. [1] Let $S=\langle G, H\rangle$ be a near permutation semigroup, where $H$ is a group. Let $G(S)=\langle G, \bar{H}\rangle$, where $\bar{H}$ is the group of permutations associated with $H$. If $2 h=2$, for all $h \in H$, then $S$ is inverse if and only if $2 \notin O_{G(S)}(1)$ and the $\operatorname{Stab}_{G(S)}(1) \subseteq \operatorname{Stab}_{G(S)}(2)$.

Theorem 4.6 gives necessary and sufficient conditions for a near permutation semigroup to be inverse. However these are somewhat technical. A consequence of the first, and simplest, of them is that the semigroup $\langle G(S), e\rangle$ is $\mathcal{R}$-unipotent. In particular, its idempotents form an $\mathcal{R}$-unipotent band. By studying the maximal subsemilattices of this band, 
and maximal inverse subsemigroups of $\langle G(S), e\rangle$, one can give alternative, and more structural, necessary and sufficient conditions for $\langle G, H\rangle$ to be inverse. This approach is contained in Chapter 4 of the author's thesis [6], and proofs will appear in [2] and [5].

Our next aim is to present a set of necessary and sufficient conditions for a semigroup $S=\langle G, H\rangle$ to be inverse and to have semilattice of idempotents $E(S)$ generated by its idempotents of rank greater than or equal to $N-1$.

Proposition 4.7. [1] Let $S$ be a semigroup with a subgroup of units $G$, a subgroup $H$ and a subsemilattice $E$ that contains the identities of $G$ and of $H$. Let also $P=\left\{x \in G \cup H: x^{-1} v x, x v x^{-1} \in E\right.$, for all $\left.v \in E\right\}$, where $x^{-1}$ denotes the group inverse of $x$ within the respective group. Then $L=\langle P, E\rangle$ is an inverse subsemigroup of $S$, with semilattice of idempotents E.

Next we obtain a necessary and sufficient condition for a semigroup $S$ to be inverse and its semilattice is generated by the idempotents of $S$ of rank greater or equal to $N-1$.

Proposition 4.8. [1] Let $S=\langle G, H\rangle$ be a near permutation semigroup, where $H$ is a group. Then $S$ is inverse with semilattice of idempotents $E(S)=\left\langle\left\{\left(\begin{array}{c}1 g \\ 2 g\end{array}\right): g \in G\right\}\right\rangle$ if and only if

i) $1 g h \in O_{G}(1)$, for all $g \in G^{\prime}$ and $h \in H$;

ii) $2 \notin O_{G(S)}(1)$;

iii) $\operatorname{Stab}_{G}(1) \subseteq \operatorname{Stab}_{G}(2)$;

iv) $1 g_{1} h g_{2}=1$ implies $2 g_{1} h g_{2}=2$, for all $g_{1} \in G^{\prime}, g_{2} \in G, h \in H_{e}$.

The following proposition describes the idempotents of rank greater than or equal to $N-2$ of an inverse near permutation semigroup $S=$ $\langle G, H\rangle$.

Proposition 4.9. [1] Let $S=\langle G, H\rangle$ be an inverse near permutation semigroup, where $H$ is a group with an identity e. Then the set of idempotents of $S$ with rank greater than or equal to $N-2$ is

$A=\left\{\left(\begin{array}{c}1 g \\ 2 g\end{array}\right): g \in G\right\} \cup\left\{\left(\begin{array}{c}1 g \\ 2 g\end{array}\right)\left(\begin{array}{c}1 g_{1} h_{1} g_{2} \\ 2 g_{1} h_{1} g_{2}\end{array}\right): g, g_{2} \in G, g_{1} \in G^{\prime}, h_{1} \in H_{e}\right\} \cup\{1\}$.

Let $S=\langle G, H\rangle$ be a near permutation semigroup. We determine necessary and sufficient conditions for $S$ to be an inverse semigroup whose semilattice of idempotents is generated by the idempotents of rank greater than or equal to $N-2$. 
Proposition 4.10. [1] Let $S=\langle G, H\rangle$ be a near permutation semigroup where $H$ is a group with identity $e$. Then $S$ is inverse, with semilattice of idempotents $E(S)$ generated by

$$
A=\left\{\left(\begin{array}{c}
1 g \\
2 g
\end{array}\right): g \in G\right\} \cup\left\{\left(\begin{array}{c}
1 g \\
2 g
\end{array}\right)\left(\begin{array}{c}
1 g_{1} h g_{2} \\
2 g_{1} h g_{2}
\end{array}\right): g, g_{2} \in G, g_{1} \in G^{\prime}, h \in H_{e}\right\} \cup\{1\}
$$

if and only if the following conditions 1), 2), 3) and 4) hold, where

1) $2 \notin O_{G(S)}(1)$;

2) $1 g_{1} h g_{2}=1$ implies $2 g_{1} h g_{2}=2$, for all $g_{1} \in G^{\prime}, g_{2} \in G, h \in H_{e}$;

3) For all $g_{1} \in G^{\prime}, g_{2} \in G, h_{1}, h_{2} \in H_{e}$, there exist $g_{3} \in G^{\prime}, g_{4} \in G, h_{3} \in$ $H_{e}$ such that $1 g_{1} h_{1} g_{2} h_{2}=1 g_{3} h_{3} g_{4}$ and $2 g_{1} h_{1} g_{2} h_{2}=2 g_{3} h_{3} g_{4}$;

4) $\operatorname{Stab}_{G}(1) \subseteq \operatorname{Stab}_{G}(2)$.

\section{Acknoledgements}

Most of the work on this paper was undertaken during the preparation of my $\mathrm{PhD}$ thesis. I am very grateful to the orientation of my supervisors Donald McAlister and Gracinda Gomes.

\section{References}

1. J.M. André, Inverse near permutation semigroups, Semigroup Forum, to appear (2004).

2. J.M. André, On subsemilattices of near permutation semigroups, in preparation (2004).

3. J.M. André, Regularity on near permutation semigroups, Comm. Algebra, to appear (2004).

4. J.M. André, Semigroups that contain all singular transformations, Semigroup Forum, to appear (2003).

5. J.M. André, The maximum inverse subsemigroup of a near permutation semigroup, in preparation (2004).

6. J.M. André, Ph. D. Thesis, University of Lisbon, 2002.

7. Robert E. Arthur, and N. Ruskuc, Presentations for two extensions of the monoid of order-preserving mappings on a finite chain, Southeast Asian Bull. Math. 24, (2000).

8. J. M. Howie, Fundamentals of Semigroup Theory, Clarendon Press, Oxford, 1995.

9. S. G. Linton, G. Pfeiffer, E. Robertson and N. Ruskuc, Gap package $P C$ software (1997).

10. K. Kearnes, A. Szendrei and J. Wood, Generating singular transformations, Semigroup Forum 63(3), 441 (2001).

11. D. B. McAlister, Semigroup for Windows PC software (1997).

12. D. B. McAlister, Semigroups generated by a group and an idempotent, Comm. Algebra 26, 515 (1998). 